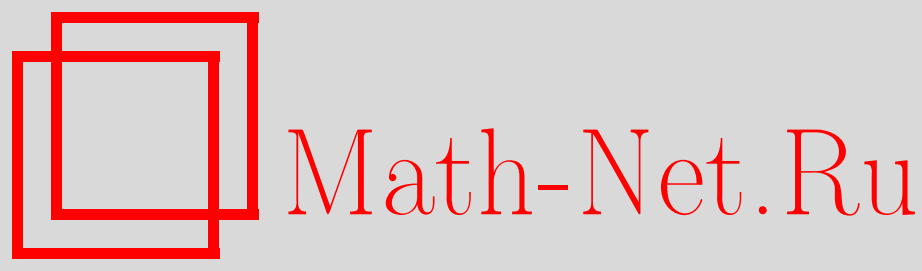

Л. А. Борисов, Ю. Н. Орлов, Анализ зависимости конечнократных аппроксимаций равновесной матрицы плотности гармонического осциллятора и функции Вигнера от правила квантования, ТМФ, 2015, том 184, номер 1, 106-116

DOI: https://doi.org/10.4213/tmf8803

Использование Общероссийского математического портала Math-Net.Ru подразумевает, что вы прочитали и согласны с пользовательским соглашением http://www.mathnet.ru/rus/agreement

Параметры загрузки:

IP: 3.89 .197 .203

26 апреля 2023 г., 18:14:50

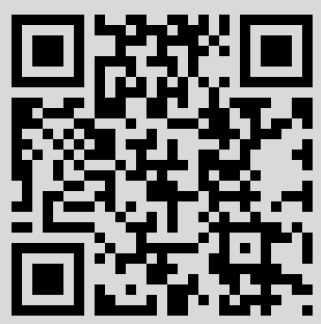




\title{
АНАЛИЗ ЗАВИСИМОСТИ КОНЕЧНОКРАТНЫХ АППРОКСИМАЦИЙ РАВНОВЕСНОЙ МАТРИЦЫ ПЛОТНОСТИ ГАРМОНИЧЕСКОГО ОСЦИЛЛЯТОРА И ФУНКЦИИ ВИГНЕРА ОТ ПРАВИЛА КВАНТОВАНИЯ
}

\begin{abstract}
Для произвольной статистической смеси $\tau$-квантований получено явное выражение для главных частей равновесной матрицы плотности и соответствующей функции Вигнера гармонического осциллятора в приближении фейнмановских аппроксимаций с использованием теоремы Чернова. На примере гамильтониана осциллятора определена скорость сходимости аппроксимации средних значений операторов наблюдаемых при увеличении порядка аппроксимаций и в зависимости от правила квантования. Показано, что сходимость аппроксимаций среднего значения оператора энергии не является равномерной по параметру Гиббса.
\end{abstract}

Ключевые слова: фейнмановские аппроксимации, теорема Чернова, правило квантования, матрица плотности, гармонический осциллятор.

DOI: $10.4213 / \operatorname{tmf} 8803$

\section{1. ВВЕДЕНИЕ}

В настоящей работе изучается скорость сходимости конечнократных приближений полугруппы Шредингера $\exp (t \widehat{H})$, где $\widehat{H}$ - эрмитов оператор Гамильтона, по формулам Фейнмана с использованием теоремы Чернова [1]. Представление полугруппы (или группы) Шредингера с помощью пределов конечнократных интегралов по декартовым степеням конфигурационного пространства классической динамической системы при стремлении кратности к бесконечности предложено Фейнманом в работах [2], [3]. В работе [4] было показано, что теорему Чернова можно обобщить для следующего представления полугруппы:

$$
\exp (t \widehat{H})=\lim _{n \rightarrow \infty} \hat{f}^{n}(t / n) .
$$

*Московский физико-технический институт, Долгопрудный, Московская обл., Россия. E-mail: leonidborisoff@gmail.com

${ }^{\dagger}$ Институт прикладной математики им. М.В. Келдыша РАН, Москва, Россия. E-mail: yuno@kiam.ru 
Здесь $\hat{f}(t)$ обозначает оператор, классическим символом которого является функция $e^{t H(q, p)}$, а предельный переход осуществляется в сильной операторной топологии. При этом классическим символом оператора Гамильтона $\widehat{H}$ является функция Гамильтона $H(q, p)$ классической системы.

Применительно к формуле (1.1) возникает вопрос о зависимости конечнократных аппроксимаций от правила квантования гамильтоновой системы. Это правило, символически обозначенное "шляпкой" в (1.1), устанавливает соответствие между классическим символом и матричным элементом квантового оператора. В настоящей работе рассматриваются линейные квантования, когда указанное соответствие представимо в виде интегрального преобразования с ядром специального вида (см. далее раздел 2).

В работе [5] с помощью формулы (1.1) было получено точное представление для конечнократной аппроксимации полугруппы $\exp (t \widehat{H})$, порожденной квантовым гамильтонианом осциллятора, для частного случая линейного квантования - так называемого $\tau$-квантования. Пример осциллятора полезен здесь тем, что он позволяет провести необходимые вычисления в явном виде, но не является тривиальным. Несмотря на то что гамильтониан квантового осциллятора $e^{t\left(q^{2}+p^{2}\right) / 2}$ не зависит от выбора квантования, квантование функции зависит от очередности действия операторов $\hat{q}$ и $\hat{p}$.

В настоящей работе найдена главная часть конечнократной аппроксимации матрицы равновесного оператора плотности $\exp (-\beta \widehat{H})$ для произвольной статистической смеси $\tau$-квантований, что обобщает результат работы [5]. Для этого используется результат работы [6] о полугруппе, эквивалентной по Чернову полугруппе, которая генерируется средним гамильтонианом. Также получено явное выражение для функции Вигнера, отвечающей аппроксимации оператора плотности. С помощью функции Вигнера найдена аппроксимация среднего значения оператора Гамильтона для осциллятора и исследована сходимость приближенного выражения к точному. Оказалось, что эта сходимость неравномерна по параметру Гиббса, и это при заданном порядке аппроксимации не позволяет представить приближенное выражение в виде суммы точного предельного значения и малого отклонения равномерно во всем диапазоне температур.

\section{2. ЛИНЕЙНОЕ КВАНТОВАНИЕ}

Пусть $\hat{A}$ - квантовый оператор, действующий в гильбертовом пространстве $L$, реализованном как пространство $L_{2}$, а $A(q, p)$ - его классический символ. Соответствие $A \rightarrow \hat{A}$ называется квантованием. Концепция линейного квантования была сформулирована в работе Березина [7], где показано, в частности, что соответствие между ядром оператора $\hat{A}$ и его классическим символом $\tilde{A}(x, y)$ определяется интегральным преобразованием с ядром $K(q, p \mid x, y)$, которое называется ядром квантования:

$$
\tilde{A}(x, y)=\int A(q, p) K(q, p \mid x, y) d q d p .
$$

При этом оператор $\hat{A}$ действует на элемент $\psi \in L$ по формуле

$$
\hat{A} \psi(x)=\int \tilde{A}(x, y) \psi(y) d y
$$


Здесь интегрирование проводится от $-\infty$ до $+\infty$. Далее пределы интегрирования для краткости не указываются, если предполагается, что интегрирование проводится по всему фазовому пространству, за исключением случаев, когда интеграл вычисляется явно.

Представителя класса линейных квантований, задаваемого ядром [8]

$$
K_{\tau}(q, p \mid x, y)=\frac{1}{2 \pi} \delta(q-(1-\tau) x-\tau y) e^{i p(x-y)}, \quad \tau \in[0,1]
$$

будем называть $\tau$-квантованием. Здесь постоянная Планка для краткости положена равной единице. Соответствующее ядро оператора, получающееся по формуле (2.1), будем обозначать как $\tilde{A}_{\tau}(x, y)$. Из формул $(2.1)$ и $(2.3)$ следует, что для $\tau$-квантования связь между ядрами операторов и их символами дается формулой

$$
\tilde{A}_{\tau}(x, y)=\frac{1}{2 \pi} \int A((1-\tau) x+\tau y, p) e^{i p(x-y)} d p .
$$

Среди $\tau$-квантований существует единственное эрмитово квантование, которое получается при $\tau=1 / 2$. В этом случае формула (2.4) определяет так называемое квантование Вейля. Другие варианты линейных эрмитовых квантований можно получить, например, с помощью линейной комбинации ядер вида (2.3), как это сделано в статье [8]. Такая комбинация может быть представлена в виде линейного интегрального преобразования ядра квантования (2.3) с некоторой обобщенной функцией $Q(\tau)$, обращающейся в ноль вне промежутка $[0,1]$ и удовлетворяющей условию

$$
\int_{0}^{1} Q(\tau) d \tau=1
$$

В результате ядро квантования и матрица оператора представляются в виде

$$
K=\int_{0}^{1} Q(\tau) K_{\tau} d \tau, \quad \tilde{A}=\int_{0}^{1} Q(\tau) \tilde{A}_{\tau} d \tau .
$$

Целью настоящей работы является нахождение зависимости конечнократных аппроксимаций матрицы плотности от правила квантования $(2.6)$, т. е. от функции $Q(\tau)$, для гармонического осциллятора, а также исследование сходимости приближенного среднего значения оператора Гамильтона к точному при увеличении порядка аппроксимаций.

Среди эрмитовых квантований общеупотребительным является квантование Вейля, но иногда используются и другие способы симметризации, поскольку нет однозначного указания на использование только какого-нибудь одного правила квантования. Приведем некоторые из них с указанием моментов $\mu_{k}$ функции симметризации,

$$
\mu_{k}=\int_{0}^{1} Q(\tau) \tau^{k} d \tau
$$

Эти моменты входят как коэффициенты перед дифференциальными операторами при квантовании классического символа в виде монома $q^{m} p^{k}$, так что квантование монома полностью определяется моментами (2.7). Перечислим следующие правила 
квантования:

$$
\begin{array}{llrl}
\text { квантование Вейля: } & Q(\tau)=\delta(\tau-1 / 2), & \mu_{k} & =\frac{1}{2^{k}} ; \\
\text { квантование Йордана: } & Q(\tau)=\frac{1}{2}(\delta(\tau)+\delta(1-\tau)), & \mu_{k} & =\frac{1}{2}, \quad k \geqslant 1 ; \\
\text { квантование Борна: } & Q(\tau)=1, & \mu_{k}=\frac{1}{k+1} .
\end{array}
$$

Отметим, что для неотрицательной функции $Q(\tau)$ формулу (2.6) можно интерпретировать как усреднение $\tau$-квантований с весом $Q(\tau)$. Но такое статистическое истолкование квантования (2.6) необязательно. Физически важно, чтобы выполнялся принцип соответствия между классическими величинами и квантовыми операторами, для чего требуется лишь выполнение условия нормировки $(2.5)$, но функция $Q(\tau)$ не обязательно должна быть неотрицательной.

\section{3. РАВНОВЕСНАЯ ФУНКЦИЯ ВИГНЕРА}

Пусть $H(q, p)$ - классический гамильтониан, а $\widehat{H}$ - отвечающий ему при квантовании с ядром (2.6) квантовый оператор Гамильтона. Равновесный оператор плотности $\hat{\rho}$ определяется формулой

$$
\hat{\rho}=\frac{1}{\operatorname{Tr} \exp (-\beta \widehat{H})} \exp (-\beta \widehat{H}) .
$$

где $\beta \in(0 ;+\infty)$ - параметр Гиббса. Обозначим через $\rho(\beta ; x, y)$ матрицу оператора плотности. Тогда среднее значение оператора $\hat{A}$ определяется по формуле

$$
\langle\hat{A}\rangle=\operatorname{Tr} \hat{\rho} \hat{A}=\int \rho(\beta ; x, y) \tilde{A}(x, y) d x d y .
$$

Если подставить в это выражение формулу (2.1) для ядра оператора $\hat{A}$, то среднее значение оператора можно получить, интегрируя его классический символ с функцией $W(\beta ; q, p)$, которая называется функцией Вигнера:

$$
\begin{gathered}
\langle\hat{A}\rangle=\int W(\beta ; q, p) A(q, p) d q d p, \\
W(\beta ; q, p)=\int \rho(\beta ; x, y) K(q, p \mid y, x) d x d y .
\end{gathered}
$$

Для $\tau$-квантования формула (3.4) имеет вид

$$
\begin{aligned}
W_{\tau}(\beta ; q, p) & =\int \rho(\beta ; x, y) K_{\tau}(q, p \mid y, x) d x d y= \\
& =\frac{1}{2 \pi} \int \rho(\beta ; q-(1-\tau) \xi, q+\tau \xi) e^{i p \xi} d \xi
\end{aligned}
$$

Из выражения (3.4) следует, что функция Вигнера явно зависит от квантования, даже если матрица плотности от него не зависит. С одной стороны, удобство представления средних значений квантовых операторов в терминах функции Вигнера состоит в том, что усреднение проводится в классическом фазовом пространстве. 
С другой стороны, помимо известного факта о неположительности (в общем случае) функции Вигнера, т. е. о невозможности ее интерпретации как плотности функции распределения, существенным затруднением при ее использовании является то, что при вычислении среднего значения квантового оператора функция Вигнера сворачивается с классическим символом, отвечающим этому оператору при том или ином квантовании. Символ же, в отличие от оператора, для произвольного квантования не восстанавливается по виду оператора, поэтому формула (3.3) оказывается практически бесполезной. Известна [7], [8] формула обращения для $\tau$-квантования (2.3):

$$
A(q, p)=\int \tilde{A}_{\tau}(q-(1-\tau) \xi, q+\tau \xi) e^{i p \xi} d \xi
$$

Для общего квантования после проведения симметризации (2.6) восстановление символа неоднозначно. Тем не менее для полиномиальных по $\hat{q}$ и $\hat{p}$ операторов можно определить символ, отвечающий квантованию с заданными моментами (2.7) функции симметризации. В этой связи пример гармонического осциллятора полезен именно тем, что он позволяет продемонстрировать указанные особенности в явном виде.

Рассмотрим классический гамильтониан

$$
H(q, p)=\frac{q^{2}}{2}+\frac{p^{2}}{2} .
$$

Соответствующий квантовый оператор не зависит от правила симметризации некоммутирующих операторов:

$$
\widehat{H}(q, p)=-\frac{1}{2} \frac{\partial^{2}}{\partial x^{2}}+\frac{x^{2}}{2} .
$$

Матрица равновесного оператора плотности (3.1) для осциллятора определяется выражением (см., например, монографию [9])

$$
\rho(\beta ; x, y)=\sqrt{\frac{\operatorname{th}(\beta / 2)}{\pi}} \exp \left(-\frac{1}{2 \operatorname{sh} \beta}\left(\left(x^{2}+y^{2}\right) \operatorname{ch} \beta-2 x y\right)\right) .
$$

Для оператора плотности с матрицей (3.9) можно получить явное выражение функции Вигнера при $\tau$-квантовании. Применяя к (3.9) преобразование (3.5), получаем

$$
\begin{aligned}
W_{\tau}(\beta ; q, p)= & \frac{1}{2 \pi} \sqrt{\frac{\operatorname{th}(\beta / 2)}{C(\beta, \tau)}} \exp \left(-i q p \frac{(1-2 \tau) \operatorname{th}(\beta / 2)}{2 C(\beta, \tau)}\right) \times \\
& \times \exp \left(-q^{2} \operatorname{th} \frac{\beta}{2} \cdot\left(1-\frac{(1-2 \tau)^{2} \operatorname{th}(\beta / 2)}{4 C(\beta, \tau)}\right)\right) \exp \left(-\frac{p^{2}}{4 C(\beta, \tau)}\right),
\end{aligned}
$$

где для краткости введено обозначение

$$
C(\beta, \tau)=\frac{1}{2} \operatorname{cth} \beta-\tau(1-\tau) \text { th } \frac{\beta}{2} .
$$

В частном случае для квантования Вейля $(\tau=1 / 2)$ имеем

$$
C(\beta, \tau)=\frac{1}{4} \operatorname{cth} \frac{\beta}{2},
$$


после чего получаем функцию Вигнера $W_{\mathrm{w}}$ для этого квантования:

$$
W_{\mathrm{W}}(\beta ; q, p)=\frac{\operatorname{th}(\beta / 2)}{\pi} e^{-\operatorname{th}(\beta / 2)\left(q^{2}+p^{2}\right)} .
$$

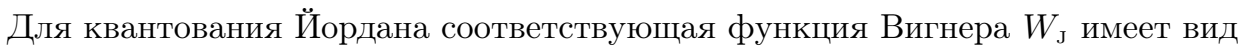

$$
W_{\mathrm{J}}(\beta ; q, p)=\frac{\sqrt{\operatorname{th}(\beta / 2) \operatorname{th} \beta}}{\pi \sqrt{2}} \exp \left(-\frac{1}{2} \operatorname{th} \beta \cdot\left(q^{2}+p^{2}\right)\right) \cos \left(p q \operatorname{th} \frac{\beta}{2} \operatorname{th} \beta\right) .
$$

При произвольном линейном квантовании с ядром (2.6) получить явное выражение для функции Вигнера затруднительно. С другой стороны, часто представляет интерес не сама функция Вигнера, а ее моменты, которые можно вычислить явно для любого линейного квантования. Рассмотрим, в частности, классический символ в виде монома $q^{2} p^{2}$. Для него среднее значение соответствующего квантового оператора зависит от квантования и при $\tau$-квантовании согласно $(3.3),(3.5)$ определяется формулой

$$
\left\langle q^{2} p^{2}\right\rangle_{\tau}=\int q^{2} p^{2} W_{\tau}(\beta ; q, p) d q d p=\frac{\operatorname{cth} \beta}{\operatorname{th}(\beta / 2)}-\frac{3}{2}+5 \tau(1-\tau) .
$$

Тогда при линейном квантовании с ядром (2.6) с учетом (2.7) получаем

$$
\left\langle q^{2} p^{2}\right\rangle=\int q^{2} p^{2} W(\beta ; q, p) d q d p=\frac{\operatorname{cth} \beta}{\operatorname{th}(\beta / 2)}-\frac{3}{2}+5 \mu_{1}-5 \mu_{2} .
$$

Средние значения такого вида требуется вычислять, например, при нахождении квадрата энергии осциллятора, для чего необходимо знать символ, отвечающий оператору

$$
\widehat{H}^{2}=\frac{1}{4} \frac{\partial^{4}}{\partial x^{4}}+\frac{x^{4}}{4}-\frac{1}{2}\left(x^{2} \frac{\partial^{2}}{\partial x^{2}}+2 x \frac{\partial}{\partial x}+1\right)
$$

при квантовании (2.6). Хотя формально процедура обращения вида (3.6) для произвольной функции симметризации $Q(\tau)$ неосуществима, в данном конкретном случае символ может иметь только полиномиальный вид, что позволяет его найти методом неопределенных коэффициентов. А именно, символ $A(q, p)$, отвечающий оператору $\hat{A}=\widehat{H}^{2}$, для эрмитовых квантований выражается через символ $H(q, p)$ оператора $\widehat{H}$ по формуле

$$
A(q, p)=H^{2}(q, p)-\frac{1}{2}+\mu_{2},
$$

где $\mu_{2}$ есть второй момент функции симметризации согласно (2.7).

Отметим, что для квантования Йордана символ, отвечающий квадрату оператора Гамильтона осциллятора, равен квадрату символа оператора Гамильтона. Но в общем случае не существует квантования, которое переводит функцию от символа в ту же функцию от оператора. Например, символ оператора $\widehat{B}=\widehat{H}^{3}$ при произвольном эрмитовом квантовании имеет вид

$$
B(q, p)=H^{3}(q, p)+\frac{7+18 \mu_{2}}{4}\left(p^{2}-q^{2}\right),
$$

так что ни при квантовании Йордана, ни при каком другом квантовании с неотрицательной мерой симметризации (2.5) его нельзя представить в виде $B(q, p)=H^{3}(q, p)$. 
Это подчеркивает нетривиальность теоремы Чернова, позволяющей обосновать процедуру построения функции от оператора через последовательность операторов от функции.

Из формулы (3.8) получаем, что средняя энергия квантового гармонического осциллятора, вычисленная с помощью функции Вигнера, не зависит от квантования и определяется выражением

$$
\langle\widehat{H}\rangle=\frac{1}{2} \operatorname{cth} \frac{\beta}{2} .
$$

В следующем разделе мы найдем приближенное среднее значение энергии по формуле (1.1) для конечнократных аппроксимаций матрицы плотности и определим скорость сходимости к точному среднему значению.

\section{4. КОНЕЧНОКРАТНЫЕ АППРОКСИМАЦИИ МАТРИЦЫ ПЛОТНОСТИ И ФУНКЦИИ ВИГНЕРА}

Для построения приближенного выражения для равновесной матрицы плотности по формуле (1.1) следует определить $n$-ю степень оператора, классическим символом которого является функция $e^{-\beta H(q, p)}$. Положим

$$
G(\beta ; x, y)=\int K(q, p \mid x, y) e^{-\beta H(q, p)} d q d p .
$$

В работе [5] было найдено выражение для $G_{\tau}(\beta ; x, y)$ в случае $\tau$-квантования:

$$
G_{\tau}(\beta ; x, y)=\frac{1}{\sqrt{2 \pi \beta}} \exp \left(-\frac{\beta}{2}((1-\tau) x+\tau y)^{2}-\frac{1}{2 \beta}(x-y)^{2}\right) .
$$

В соответствии с формулой (1.1) теперь надо заменить в (4.2) $\beta$ на $\beta / n$ и определить $n$-ю степень такого оператора. Точное выражение для $n$-й степени оператора (4.2) в случае $\tau$-квантования было найдено в работе [5] в явном виде. Матрица соответствующего оператора имеет экспоненциальный вид, и в показателе экспоненты можно выделить главную часть по $1 / n$ :

$$
\begin{aligned}
G_{\tau}^{n}\left(\frac{\beta}{n} ; x, y\right)= & \int K_{\tau}\left(q_{1}, p_{1} \mid x, z_{1}\right) \ldots K_{\tau}\left(q_{n}, p_{n} \mid z_{n-1}, y\right) \times \\
& \times \exp \left(-\frac{\beta}{n} \sum_{k=1}^{n} H\left(q_{k}, p_{k}\right)\right) d q d p d z= \\
= & \frac{1}{\sqrt{2 \pi \operatorname{sh} \beta}} \exp \left(-\frac{x^{2}+y^{2}}{2}\left(\operatorname{cth} \beta+\frac{\beta}{n \operatorname{sh}^{2} \beta}\right)\right) \times \\
& \times \exp \left(\frac{x y}{\operatorname{sh} \beta}+\frac{\beta\left(1+\tau-2 \tau^{2}\right) x^{2}}{2 n}+\frac{\beta \tau(3-2 \tau) y^{2}}{2 n}+o\left(\frac{1}{n}\right)\right) .
\end{aligned}
$$

Из данного выражения следует, что зависимость этого оператора от параметра $\tau$ в процессе взятия предела при $n \rightarrow \infty$ исчезает, и в результате с точностью до нормировочного множителя получается матрица плотности (3.9) гармонического осциллятора, которая не зависит от правила квантования. 
Если теперь в формуле (4.3) заменить ядро $\tau$-квантования (2.3) на более общее (2.6), то вычислить ядро оператора $G^{n}(\beta / n ; x, y)$ в явном виде не удается. Но с точностью $O(1 / n)$ показатель экспоненты в формуле, аналогичной (4.3), может быть определен. Для этого заметим, что квантование (2.6) представляет собой статистическую смесь $\tau$-квантований, а получающийся в результате такой смеси гамильтониан $\widehat{H}=\int Q(\tau) \widehat{H}_{\tau} d \tau$ является результатом усреднения гамильтонианов, получающихся с помощью разных $\tau$-квантований. В работе [6] была доказана теорема о том, что при достаточно общих условиях на операторы (если средний оператор замыкаем и является генератором сильно непрерывной полугруппы) среднее значение полугруппы эквивалентно по Чернову полугруппе, генерируемой средним гамильтонианом. Эта теорема была доказана в [6] применительно к усреднению различных классических гамильтонианов, генерирующих одинаковые траектории в классическом фазовом пространстве, но порождающих разные квантовые операторы. Однако эта теорема может быть применена и непосредственно к усреднению квантовых операторов.

Напомним [10], что операторнозначая функция $\hat{f}(t)$, действующая из некоторой правой полуокрестности нуля на числовой оси в банахово пространство $B(X)$ линейных ограниченных операторов, действующих в банаховом пространстве $X$, называется эквивалентной по Чернову полугруппе $\hat{g}(t)$, если для всех $T>0$ и $u \in X$ выполняется условие

$$
\lim _{n \rightarrow \infty} \sup _{t \in[0 ; T]}\left\|\left(\hat{f}^{n}(t / n)-\hat{g}(t)\right) u\right\|_{X}=0 .
$$

В результате оказывается возможным провести усреднение в смысле эквивалентности по Чернову в экспоненте оператора (4.3). Для линейного квантования (2.6) имеем

$$
\begin{aligned}
& G^{n}\left(\frac{\beta}{n} ; x, y\right)=\int K\left(q_{1}, p_{1} \mid x, z_{1}\right) \ldots K\left(q_{n}, p_{n} \mid z_{n-1}, y\right) \times \\
& \quad \times \exp \left(-\frac{\beta}{2 n} \sum_{k=1}^{n}\left(q_{k}^{2}+p_{k}^{2}\right)\right) d q d p d z= \\
& =\int Q\left(\tau_{1}\right) \ldots Q\left(\tau_{n}\right) G_{\tau_{1}}\left(\frac{\beta}{n} ; x, z_{1}\right) \ldots G_{\tau_{n}}\left(\frac{\beta}{n} ; z_{n-1}, y\right) d z_{1} \ldots d z_{n-1} d \tau_{1} d \tau_{n} .
\end{aligned}
$$

Представим ядро (4.2) оператора, которому отвечает символ $e^{-\beta H}$, в виде

$$
\begin{aligned}
& G\left(\frac{\beta}{n} ; x, y\right)=\frac{1}{\sqrt{2 \pi \beta}} \exp \left(-\frac{n(x-y)^{2}}{2 \beta}\right) \times \\
& \quad \times \exp \left(-\frac{\beta}{2 n}\left((1-\tau)^{2} x^{2}+\tau^{2} y^{2}+2 x y \tau(1-\tau)\right)\right)= \\
& =\frac{1}{\sqrt{2 \pi \beta}} \exp \left(-\frac{n(x-y)^{2}}{2 \beta}\right)\left(1-\frac{\beta}{2 n}\left((1-\tau)^{2} x^{2}+\tau^{2} y^{2}+2 x y \tau(1-\tau)\right)+o\left(\frac{1}{n}\right)\right),
\end{aligned}
$$


так что

$$
\begin{aligned}
G\left(\frac{\beta}{n} ; x, y\right)= & \int_{0}^{1} Q(\tau) G_{\tau}\left(\frac{\beta}{n} ; x, y\right) d \tau=\frac{1}{\sqrt{2 \pi \beta}} \exp \left(-\frac{n(x-y)^{2}}{2 \beta}\right) \times \\
& \times\left(1-\frac{\beta}{2 n}\left(\left(1-2 \mu_{1}+\mu_{2}\right) x^{2}+\mu_{2} y^{2}+2 x y\left(\mu_{1}-\mu_{2}\right)\right)+o\left(\frac{1}{n}\right)\right)= \\
= & \frac{1}{\sqrt{2 \pi \beta}} \exp \left(-\left(\frac{n}{2 \beta}+\frac{\beta \mu_{2}}{2 n}\right)(x-y)^{2}+\left(1-2 \mu_{1}\right) x^{2}+2 x y \mu_{1}+o\left(\frac{1}{n}\right)\right) .
\end{aligned}
$$

Последнее выражение в цепочке равенств является следствием вышеупомянутой теоремы об эквивалентности по Чернову полугруппы, порождающейся средним оператором Гамильтона, среднему значению полугрупп, каждая из которых порождена отдельным $\tau$-проквантованным гамильтонианом. Подставляя полученное выражение в (4.4) в качестве результата интегрирования по $\tau$ каждого из содержащихся там сомножителей, получаем выражение для эквивалентного по Чернову оператора плотности $n$-кратной аппроксимации:

$$
\begin{aligned}
& G^{n}\left(\frac{\beta}{n} ; x, y\right)=\frac{1}{\sqrt{2 \pi \operatorname{sh} \beta}} \exp \left(-\frac{x^{2}+y^{2}}{2} \operatorname{cth} \beta+\frac{x y}{\operatorname{sh} \beta}\right) \times \\
& \quad \times \exp \left(-\frac{\left(x^{2}+y^{2}\right) \beta}{2 n \operatorname{sh}^{2} \beta}+\frac{\beta x^{2}}{2 n}\left(1+\mu_{1}-2 \mu_{2}\right)+\frac{\beta y^{2}}{2 n}\left(3 \mu_{1}-2 \mu_{2}\right)+o\left(\frac{1}{n}\right)\right) .
\end{aligned}
$$

Для эрмитовых квантований $\mu_{1}=1 / 2$, и из (4.5) получаем в этом случае

$$
\begin{aligned}
& G^{n}\left(\frac{\beta}{n} ; x, y\right)=\frac{1}{\sqrt{2 \pi \operatorname{sh} \beta}} \times \\
& \quad \times \exp \left(-\frac{x^{2}+y^{2}}{2}\left(\operatorname{cth} \beta+\frac{\beta}{n}\left(\frac{1}{\operatorname{sh}^{2} \beta}+\frac{3}{2}-2 \mu_{2}\right)\right)+\frac{x y}{\operatorname{sh} \beta}+o\left(\frac{1}{n}\right)\right) .
\end{aligned}
$$

Далее мы рассматриваем эрмитовы квантования.

Из формулы (4.6) следует, что главная часть оператора $\widehat{G}^{n}$ по $1 / n$ зависит от квантования только через второй момент функции симметризации. Для квантования Вейля он минимален (см. выше раздел 2), а для квантования Йордана максимален, что позволяет оценить сверху неточность приближенного вычисления матрицы плотности для произвольного эрмитового квантования: для разных квантований относительное различие между матрицами плотности не превосходит величины $1-e^{-(\beta / 4 n)\left(x^{2}+y^{2}\right)}$.

Нормированная равновесная матрица плотности гармонического осциллятора в $n$-м приближении формулы (1.1) для линейного квантования с ядром (2.6) имеет вид

$$
\begin{aligned}
\rho_{n}(\beta ; x, y)= & \sqrt{\frac{\operatorname{th}(\beta / 2)+\lambda_{n}(\beta)}{\pi}} \times \\
& \times \exp \left(-\frac{1}{2 \operatorname{sh} \beta}\left(\left(x^{2}+y^{2}\right)\left(\operatorname{ch} \beta+\lambda_{n}(\beta) \operatorname{sh} \beta\right)-2 x y\right)\right),
\end{aligned}
$$

где величина $\lambda_{n}(\beta)$ имеет порядок $1 / n$ :

$$
\lambda_{n}(\beta)=\frac{\beta}{n}\left(\frac{1}{\operatorname{sh}^{2} \beta}+\frac{3}{2}-2 \mu_{2}\right)+o\left(\frac{1}{n}\right) .
$$


Приближенная матрица плотности (4.7) отличается от точной (3.9) величиной $\lambda_{n}(\beta)$. Эта величина положительна при всех ненулевых $\beta$, так как $\mu_{2} \in[1 / 4,1 / 2]$.

Следующий шаг состоит в нахождении функции Вигнера $W_{n, \tau}(\beta ; q, p)$, которая отвечает матрице плотности (4.7) при $\tau$-квантовании. Хотя, как уже говорилось, для общего квантования (2.6) функцию Вигнера явно вычислить не удается, ее вид для $\tau$-квантования необходим, чтобы найти средние значения операторов $\left\langle\hat{A}_{n}\right\rangle$ в приближении $n$-го порядка аппроксимации матрицы плотности для общего квантования. Вид функции Вигнера близок к выражению (3.10), только теперь в нем содержится поправка $\lambda_{n}$ из (4.7). Имеем

$$
\begin{aligned}
& W_{n, \tau}(\beta ; q, p)=\int \rho_{n}(\beta ; x, y) K_{\tau}(q, p \mid x, y) d x d y= \\
& =\frac{1}{2 \pi} \sqrt{\frac{Z_{n}(\beta, \tau)}{C_{n}(\beta, \tau)}} \exp \left(-i q p \frac{(1-2 \tau) Z_{n}(\beta, \tau)}{2 C_{n}(\beta, \tau)}\right) \times \\
& \quad \times \exp \left(-q^{2} Z_{n}(\beta, \tau)\left(1-\frac{(1-2 \tau)^{2} Z_{n}(\beta, \tau)}{4 C_{n}(\beta, \tau)}\right)\right) \exp \left(-\frac{p^{2}}{4 C_{n}(\beta, \tau)}\right) .
\end{aligned}
$$

Здесь для краткости введены обозначения

$$
C_{n}(\beta, \tau)=C(\beta, \tau)+\frac{\lambda_{n}}{2}(1-2 \tau(1-\tau)), \quad Z_{n}(\beta, \tau)=\operatorname{th} \frac{\beta}{2}+\lambda_{n},
$$

причем $C(\beta, \tau)$ - та же функция, что и в $(3.10)$.

Принципиальным отличием приближенного выражения (4.8) от точного (3.10) является то, что функция Вигнера в $n$-м приближении несимметрична по $q$ и $p$ даже для эрмитовых квантований, так как из-за добавки $\lambda_{n}$ получается, что $4 C_{n} \neq 1 / Z_{n}$, и лишь при $n \rightarrow \infty$ симметрия восстанавливается. Это обстоятельство приводит к нетривиальным эффектам при вычислении средних значений операторов по приближенной функции Вигнера. Среднее значение $\langle\hat{A}\rangle_{n}$ квантового оператора $\hat{A}$ в $n$-м порядке приближения для общего квантования (2.6) определяется формулой

$$
\langle\hat{A}\rangle_{n}=\int_{0}^{1} Q(\tau) \int W_{n, \tau}(\beta ; q, p) A(q, p) d p d q d \tau .
$$

Для средней энергии осциллятора в $n$-м приближении получаем выражение, зависящее от квантования:

$$
\langle\widehat{H}\rangle_{n}=\frac{\operatorname{cth}(\beta / 2)}{4}\left(1+\frac{1}{1+\lambda_{n} \operatorname{cth}(\beta / 2)}\right)+\frac{\lambda_{n}}{2}\left(1-\mu_{2}\right) .
$$

Как видно, средняя энергия нелинейно зависит от $\lambda_{n}$, причем разлагать выражение (4.10), приближенную матрицу плотности или функцию Вигнера в ряд по $1 / n$ с точностью до первого порядка не следует, так как это может привести к отрицательным значениям средней энергии, в отличие от формулы (4.10). Это связано с тем, что как при малых $\beta$ (высокие температуры, квазиклассическое поведение), так и при больших $\beta$ (малые температуры, квантовое поведение) сходимость в (4.10) при $n \rightarrow \infty$ неравномерна по параметру $\beta$. Действительно, с учетом явного вида величины $\lambda_{n}$ из (4.7) следует, что при малых $\beta$ мы имеем $\lambda_{n} \approx 1 / n \beta$, а при больших $\beta$ получаем $\lambda_{n} \approx \beta / n$. 
Таким образом, мы показали, что функция Вигнера, отвечающая конечнократной аппроксимации матрицы плотности по формуле Фейнмана для гармонического осциллятора, сходится к точной функции Вигнера и скорость сходимости имеет порядок $1 / n$. Сходимость не является равномерной по параметру Гиббса $\beta$ как при $\beta \rightarrow 0$, так и при $\beta \rightarrow \infty$. Тот же вывод справедлив и для моментов приближенной матрицы плотности или функции Вигнера.

Зависимость от квантования для аппроксимаций матрицы плотности и средних значений операторов проявляется в порядке $1 / n$ и не приводит к качественным эффектам относительно типа и скорости сходимости рассматриваемых приближений.

Подчеркнем, что к практическому использованию конечнократных фейнмановских аппроксимаций как численной процедуре следует подходить очень аккуратно. Предполагая использовать для матрицы плотности некоторой квантовой системы вместо точного выражения, которое может быть неизвестно, его аппроксимацию, получающуюся численно при фиксированном порядке приближения $n$, следует также определить допустимый промежуток изменения параметра $\beta$, внутри которого аппроксимация достаточно близка к точному значению. Как показывает разобранный в настоящей работе пример, выделение главной линейной части не только в приближенном выражении для матрицы плотности, но и в выражениях для ее моментов может приводить к ошибочным численным значениям, если проводить расчеты при некотором заданном $n$ во всем диапазоне температур.

\section{Список литературы}

[1] P. R. Chernoff, J. Funct. Anal., 2:2 (1968), 238-242.

[2] R. P. Feynman, Rev. Modern Phys., 20:2 (1948), 367-387.

[3] R. P. Feynman, Phys. Rev., 84:1 (1951), 108-128.

[4] O. G. Smolyanov, A. G. Tokarev, A. Truman, J. Math. Phys., 43:10 (2002), 5161-5171.

[5] Ю. Н. Орлов, В. Ж. Сакбаев, О. Г. Смолянов, ТМФ, 172:1 (2012), 122-137.

[6] Ю. Н. Орлов, В. Ж. Сакбаев, О. Г. Смолянов, Тр. МИАН, 285 (2014), 232-243.

[7] Ф. А. Березин, ТМФ, 6:2 (1971), 194-212.

[8] Ю.Н. Орлов, Основы квантования вырожденных динамических систем, МФТИ, М., 2004.

[9] Р. Фейнман, Статистическая механика, Мир, М., 1975.

[10] O. G. Smolyanov, H. Weizsäcker, O. Wittih, Potential Anal., 26:1 (2007), 1-29. 